\title{
CENTRAL CONTROL COMPONENTS OF A "SIMPLE" STRETCH REFLEX
}

\author{
F. CLARAC ${ }^{1}$, D. CATTAERT ${ }^{2}$ and D. LE RAY ${ }^{1}$
}

${ }^{1}$ Laboratoire de Neurobiologie et Mouvements, UPR 9011 du Centre National de la Recherche Scientifique, Institut Fédératif de Recherche "Sciences du Cerveau", 31 Chemin Joseph Aiguier, 13402 Marseille Cedex 20, France.

${ }^{2}$ Laboratoire de Neurobiologie des Réseaux, UMR 5816 du Centre National de la Recherche Scientifique, Université de Bordeaux 1, Biologie animale, Bâtiment B2, Avenue des Facultés, 33405 Talence cedex, France.

Key Words: motor control, stretch reflex, presynaptic inhibition, reflex modulation, CPG, locomotion, invertebrate, crayfish.

Correspondence to: François CLARAC, address is above. 


\section{SUMMARY}

The monosynaptic stretch reflex is a fundamental feature of sensory-motor organization in most animal groups. In isolation, it serves largely as a negative feedback devoted to postural controls. However, when it is involved in diverse movements, it can be deeply modified by central command circuits. In order to understand the implications of such modifications, we have focused on a model system which has been studied at many different levels: the crayfish walking system. In this short review, we give an overview of a sensory-motor network present in this system, examining all the central influences that are able to reorganize the sensory inputs, and comparing the findings in this system to those of other species. Recent studies have revealed several levels of control and modulation (such as the sensory afferent, the output synapse from the sensory afferent, and the membrane properties of the post-synaptic neuron) that operate complex and highly adaptive sensory-motor processing. During the unfolding of a given motor task, such mechanisms reshape completely the sensory message, such that the stretch reflex becomes a part of the central motor command. 


\section{Introduction}

Among the sensory-motor loops that have been described, the stretch reflex illustrates the simplest and the most widespread proprioceptive feedback system in both vertebrates ${ }^{1}$ and invertebrates (called the resistance reflex in this group ${ }^{2-4}$ ): when a muscle is stretched, sensory feedback causes an activation of the motoneurons (MNs) innervating that muscle. Functionally, this postural negative feedback would help in maintaining a given position. For the past decade, this reflex has been studied in a number of vertebrate and invertebrate species. Recent information has shown that the reflex can be modulated at different locations within the neural circuits. Indeed, during the past decade a number of studies have pointed out the relationship between sensory-motor pathways and centrally generated rhythmic motor activities (for a review see 5). The concept of a central pattern generator (CPG) was initially proposed to describe populations of neurons that elaborate a basic rhythmic motor pattern activity in the absence of any movement related sensory feedback. First demonstrated in locust ${ }^{6}$, CPGs have subsequently been described in many invertebrate and vertebrate models (for a review see 7). In addition to controlling motor output, CPGs also exert control over sensory-motor pathways in many systems. For example, during rhythmic motor activities such as walking, the "stretch" reflex is modulated not only in intensity, but also in sign (for a

review see 8). Finally, long term changes have recently been described in this reflex ${ }^{9,10}$, making it a possible target for motor learning. A striking feature of these studies is that the central control of this reflex appears to share common properties in different vertebrate and invertebrate animal models.

For more than ten years, we have been studying the stretch reflex in the crayfish walking system, and we have accumulated a wealth of information that illustrates all the mechanisms, from the cellular to the network level, mentioned above, with the advantage that they were all studied in the same circuit, mostly on identified neurons. Therefore, in this short review, we present an overview of all these central mechanisms that control this reflex in the crayfish walking system, and we compare these findings to those described in other systems. This review will analyze how these various mechanisms operate simultaneously or alternately in different states of the locomotor system, to ensure different behavioral functions. 
Modulation of the stretch reflex will be considered successively at 3 levels, in sensory afferents, in interneuronal pathways and in MNs. In addition to these immediate regulating mechanisms, long-term modifications will be also considered in the last part of this short review.

\section{Organization of the "stretch" reflex}

The stretch reflex is a negative feedback system based on proprioceptors that detect and code for the geometry and changes in position of joints. Although vertebrates, insects and crustaceans use different types of proprioceptors (Fig. 1), this negative feedback system obeys similar principles : proprioceptive organs, lying in parallel with skeletal muscles, activate muscles that counteract the imposed movement.

In vertebrates, the proprioceptors involved in the reflex are the muscle spindles (Fig. 1A), which lie within skeletal muscles, parallel to the muscle fibers, where they respond to stretch of the muscle. Muscle spindles consist of intrafusal fibers surrounded in their central region by two types of sensory endings (primary and secondary). There is usually just one primary ending in each spindle, consisting of a single group Ia afferent axon, and just one secondary ending consisting of the branches of a single group II afferent axon. The group Ia afferent neurons monosynaptically excite the MNs of the same muscle ${ }^{1}$ (Fig. 1A).

In insects and crustaceans, the proprioceptors involved in negative feedback are mainly chordotonal organs (Fig. 1B), consisting of an elastic strand that crosses the joint. There is often just one chordotonal organ in each joint, located outside the skeletal muscles but parallel to one of them (see Box 1). In the elastic strand of the chordotonal organ, tens of sensory neurons coding for the different parameters of positions and movements are divided in two functionally distinct groups: some respond to stretch, and others respond to release. However, as is the case for vertebrate Ia afferents, each of these sensory groups monosynaptically excites the MNs of the muscle that counteract the imposed movement ${ }^{2,4}$ (Fig. 1B).

In vertebrates, the contractile polar regions of intrafusal fibers are innervated by gamma MNs. When activated, these gamma MNs change the sensitivity of the sensory endings to stretch (for a review see 11). In arthropods, a similar arrangement exists in one 
type of proprioceptive organ, the muscle receptor organ (MRO); however, in contrast to chordotonal organs, muscle receptor organs are not present in all leg joints ${ }^{12}$. Although this type of control is also important at some joints, this short review will not consider efferent control of proprioceptors.

The direct convergence of sensory fibers onto each $\mathrm{MN}$ is a shared feature of the resistance reflex in arthropods (insects ${ }^{2}$, crustacea $^{3,4}$ ) and the stretch reflex in vertebrates ${ }^{13}$. For example, in crayfish, where 8 of the 12 depressor MNs are involved in the stretch reflex, each of these MNs is monosynaptically excited by 2 to 5 of the 20 proprioceptive neurons from the coxo-basipodite chordotonal organ ( $\mathrm{CBCO}$ ) that respond to levation of the basipodite (Fig. 2). A much larger proportion of MNs (up to 100\%) seem to be involved in the stretch reflex in the cat spinal cord, where a substantial number of single Ia afferent fibers have been demonstrated to project onto most (65-80\%) of the homonymous $\mathrm{MNs}^{14}$. However, the details of the connections are better known in crayfish, in which all the MNs innervating a given muscle can be successively recorded in the same experiment. In this case, it appears that, in addition to convergence of multiple sensory fibers onto each $\mathrm{MN}$, there is also considerable, but highly heterogeneous, divergence of sensory neurons onto the MNs. A single $\mathrm{CBCO}$ fiber ${ }^{15}$, for example, may project onto from 1 to $8 \mathrm{MNs}$.

\section{Other components of the sensory-motor system}

Although most of the sensory-motor connections are mediated by classical chemical synapses, electrical coupling has also been described in some sensory-motor connections, at least in some animal groups. For example, in crustacea, in the thoraco-coxal muscle receptor organ (TCMRO), a proprioceptive structure that codes movements and positions of the first leg joint, the two sensory fibers are electrically coupled to $\mathrm{MNs}^{16}$ (see Fig. 4E). Similar electrical connections also exist between Ia afferents and MNs in the frog 17 .

In parallel with monosynaptic chemical and electrical connections, the negative feedback reflex also involves polysynaptic pathways through spiking interneurons (INs). In 
vertebrates, a disynaptic pathway involving Ia inhibitory INs inhibits the antagonist MNs and is responsible for the reciprocal innervation described by Sherrington (see Fig. 1A). In addition, recent studies suggest that muscle spindle afferents may activate the excitatory interneurons of disynaptic pathways to homonymous MNs, and activate other INs involved in the half-center responsible for locomotion ${ }^{5}$ (see below). In arthropods, polysynaptic pathways also involve non-spiking INs which are the premotor elements controlling MN activity in locust $^{2,18}$ (see Fig. 4A) and in stick insects ${ }^{19,20}$ (see Fig. 4B). Relative to that of vertebrates, the wiring of the reciprocal innervation demonstrated in the locust ${ }^{21}$ is more complex, and involves both spiking and non-spiking INs (see Fig. 4A).

The polysynaptic pathways are partially responsible for the fact that the negative feedback reflex is not rigid in arthropods ${ }^{22}$ and vertebrates ${ }^{23}$. Indeed, this reflex can be modified not only in intensity but also in sign, when the resistance (negative feedback) reflex is reversed into an assistance (positive feedback) reflex. This reflex reversal involves both presynaptic inhibition of primary afferents in both vertebrates ${ }^{24}$ and invertebrates ${ }^{25}$, and changes in the activation of spiking and non-spiking interneurons of the polysynaptic pathways in insects $^{26}$ and crustacea ${ }^{27}$. 


\section{The in vitro preparation of the crayfish locomotor network.}

Unlike vertebrates, in which proprioceptive coding of movements is mediated by stretch sensitive fusorial organs inserted into each antagonistic muscle (Fig. 1A), a single proprioceptor (chordotonal organ) codes for both directions of angular joint movement in crustaceans 28 (Fig. 1B). At the base of the leg, the second joint (coxo-basipodite, $\mathrm{CB}$ ) is responsible for the upward and downward movements (Fig. 2A) driven by levator (LEV) and depressor (DEP) muscles, respectively (Fig. 2B). This joint, which is involved in both the swing and the stance phases, is essential for walking activity of the animal. The $\mathrm{CB}$ chordotonal organ $(\mathrm{CBCO})$ is composed of an elastic strand that crosses the $\mathrm{CB}$ joint (Fig. 1B). Sensory bipolar cells are inserted in this strand. Some are sensitive to the stretch of the strand, which occurs during downward leg movements, as the levator muscles are stretched. Others are sensitive to the release of the strand, which occurs during upward leg movements, as the depressor muscles are stretched.

An in vitro preparation of the central nervous system (Fig. 2C) commanding leg movements has been developed ${ }^{4}$. In this preparation, the $\mathrm{CBCO}$ is dissected and pinned out in a Petri dish in such a position that a mechanical puller attached to the distal end of the $\mathrm{CBCO}$ imposes stretches and releases on the strand in exactly the same way as in the intact leg during locomotion. Using this preparation, which allows paired intracellular recordings from identified MNs and CBCO sensory terminals (Fig. 2D, $1 \mathrm{E})$, it is possible to record intracellularly from sensory neurons, and to study the monosynaptic connections they make with identified MNs (Fig. 2E).

The network studied using this in vitro preparation consists of about 80 neurons: the CBCO is composed of 40 bipolar cells, and the output consists of 12 depressor MNs and 19 levator MNs. In addition, a dozen or so INs are involved in polysynaptic pathways between CBCO neurons and these MNs. Among CBCO sensory neurons, twenty respond when the strand is stretched, and the remaining twenty respond when the strand is released. The reflex responses to imposed leg movements can be studied either when the network is in a tonic state (negative feedback; Fig. 2F), or during rhythmic activity induced by oxotremorine, a muscarinic agonist of acetylcholine 29 . Moreover, by using a high $\mathrm{Ca}^{2+}$ and high $\mathrm{Mg}^{2+}$ saline that raises the threshold for spiking, monosynaptic sensory-motor connections can be specifically studied $^{30}$ (Fig. 2E, 2F). 


\section{The sensory terminal as a sensory-processing structure}

It has been known for many years that the afferent message can be modified by presynaptic inhibition within sensory axons in vertebrates 31 . More recent results indicate that, at least in crustaceans, two other mechanisms may modify the function of sensory neurons : (i) the existence of electrical synapses between sensory afferent terminals ${ }^{32}$, and (ii) the possibility of modifying the sensitivity of proprioceptive neurons by neuromodulatory substances 33,34 .

Nearly 40 years ago, primary afferents were shown to be the site of presynaptic modulation in both vertebrates and invertebrates. In 1957, Frank \& Fuortes ${ }^{31}$ reported the first evidence of presynaptic inhibition in cat group I afferent fibers. During fictive locomotion in the cat, group I afferent fibers display rhythmic bursts of PADs (primary afferent depolarizations) phase-locked with the locomotor rhythm ${ }^{35}$. Similar PADs of central origin have been reported in crayfish. In insects, in addition to centrally originating PADs, chordotonal afferents are presynaptically inhibited by PADs generated by other sensory afferents of the same sense organ ${ }^{36}$. However, such presynaptic inhibition of sensory origin has never been observed in chordotonal terminals of the crayfish. Recent studies have demonstrated that the mechanisms that underlie PADs are similar in vertebrates and invertebrates $^{24,25,37,38}$ (with some specific differences in the case of insects, see below). However, in spite of the similarities in the mechanisms underlying PADs, there are differences in the mechanisms by which the PADs produce presynaptic inhibition.

Recent studies in crayfish have provided new insight into the mechanisms of presynaptic inhibition ${ }^{39}$. During oxotremorine-induced rhythmic activity, intracellular recordings from $\mathrm{CBCO}$ terminals in the thoracic ganglion commanding the corresponding leg revealed the presence of PADs occurring in phase with rhythmic depressor motor bursts (Fig. 3A). PADs were demonstrated to be inhibitory in experiments involving simultaneous intracellular recordings from a CBCO terminal and a postsynaptic MN (Fig. 3B). When PADs occurred, the amplitude of both the orthodromic spike and the corresponding EPSP in the 
postsynaptic $\mathrm{MN}$ were reduced proportionally to PAD amplitude ${ }^{25}$. Similar effects were reproduced by direct pressure-application of GABA onto the $\mathrm{CBCO}$ terminals ${ }^{25}$. PAD-mediated presynaptic inhibition in crayfish $\mathrm{CBCO}$ afferents is produced by specialized INs (the PADIs - not yet identified) using GABA as their neurotransmitter, and activating a $\mathrm{GABA}_{\mathrm{A}}$-like receptor ${ }^{40}$ associated with a chloride conductance whose reversal potential is around $-35 \mathrm{mV}$ (which explains why PADs are depolarizing). The PADs in cat primary afferent neurons appear to involve the same mechanisms: the depolarizations are mediated by GABA, and involve chloride conductances with similar reversal potentials ${ }^{41}$. In contrast, the reversal potential for chloride is much closer to the resting membrane potential ${ }^{38}$ in insect sensory neurons, and therefore the amplitude of PADs never exceed a few $\mathrm{mV}$. The mechanisms of GABA-mediated presynaptic inhibition are therefore likely different in the three groups. Whereas in insects it likely results from a shunting effect, in cat, due to the large amplitude of PADs, inactivation of sodium channels would likely play the major role ${ }^{42}$. Crayfish would represent an intermediate situation in which small PADs are exclusively shunting, while large ones exert both shunting effect and inactivation of sodium channels ${ }^{39}$.

In addition to these two mechanisms (shunting and inactivation of sodium channels), a third mechanism, acting at the level of the sensory neuron, seems to be involved in the phasic modulation of the reflex in both cat and crayfish. Due to the very depolarized value of the equilibrium potential for chloride, the amplitude of rhythmic PAD bursts may be large enough to elicit antidromic spikes during rhythmic motor activity in cat ${ }^{35}$ and in crayfish ${ }^{43}$. The analysis of antidromic discharges in $\mathrm{CBCO}$ neurons has demonstrated that they were directly related to the GABA-evoked increase of chloride conductance ${ }^{43}$. A recent study demonstrated that antidromic discharges exert a powerful direct inhibition on peripheral sensory coding by $\mathrm{CBCO}$ neurons at the site of mechanotransduction itself ${ }^{44}$. Increasing the frequency and the duration of the antidromic burst results in a reduction in frequency of the sensory discharge observed in CBCO neurons that code for position. At high frequency $(50-100 \mathrm{~Hz})$, antidromic trains generally result in the cessation of sensory input activity that may outlast the antidromic train for up to $500 \mathrm{~ms}$. Thus, depending on the level of activation of the chloride channel associated with the $\mathrm{GABA}_{\mathrm{A}}$ type receptor in their primary afferents, crayfish and possibly 
vertebrates can modulate the proprioceptive message in three ways : (i) small PADs would finely adjust the level of synaptic transmission of the sensory message to the MNs by a local shunting mechanism (see Fig. 2B); (ii) larger PADs would inactivate the sodium channel over a large distance ${ }^{42}$ and therefore result in a complete blocking of the transmission of the proprioceptive signal; (iii) during larger amplitude PADs, antidromic bursts would block the sensory activity of the proprioceptive neurons for a longer duration. The latter two effects would prevent co-contraction of antagonistic muscles during "active" programmed movements.

In addition to presynaptic inhibition, proprioceptive afferents in crayfish may use electrical connections between sensory fibers ${ }^{32}$ to achieve complex data processing. Confocal analysis of dye-coupled axons has revealed the existence of large zones of close appositions between Lucifer Yellow-stained axons (Fig. 3C). By increasing the amount of transmitter released $^{32}$, weak electrical coupling (Fig. 3D) serves to enhance transmission of sensory signal from of afferents sharing the same coding properties, onto depressor MNs. Stronger electrical coupling may induce spike triggering in the coupled $\mathrm{CBCO}$ afferent, resulting in an increased number of active afferents. This mechanism could act as a coincidence detector ${ }^{45,46}$ and result in a more precise and effective resistance reflex.

Neuromodulation constitutes a third level of control of the proprioceptive message. This category of modulatory mechanisms is generally achieved by neuroactive substances that are either released by neurons, or are present in the blood. In the presence of such substances, the properties of the neurons are changed (gating effects). Sometimes, the changes persist after the neuroactive substance has been removed (trigger effects). Modulatory effects on sensory neurons were first described in the lobster oval organ ${ }^{33}$ (sensory element of the ventilatory system): while the monoamine serotonin (5HT) inhibits the firing activity of this mechanoreceptor, octopamine and proctolin enhance it. Similarly, in crayfish, 5HT modulates both sensory coding ${ }^{34}$ and reciprocal inhibition between antagonistic $\mathrm{MNs}^{47}$. In fact, 5HT exerts a dual effect on the coding characteristics of CBCO sensory neurons that depends on its concentration. At low concentrations $\left(10^{-9}\right.$ to $10^{-6} \mathrm{M}$ ), the sensory neurons (phaso-tonic and phasic) increase their discharge (Fig. 3E), resulting in an enhancement of the stretch reflex. 
However, at higher doses $\left(10^{-4} \mathrm{M}\right)$, the effect of $5 \mathrm{HT}$ is reversed, and the discharge frequency diminishes $^{34}$. The mechanisms by which 5HT modifies the coding of movement parameters are still unknown. Note that neuromodulatory effects may affect only specific sensory-motor pathways. For example, in the stick insect, octopamine inhibits the resistance reflex pathways, but does not affect positive feedback in active animals ${ }^{48}$; in the locust, octopamine enhances the position (tonic) component response of the femur chordotonal organ, but not the movement (phasic) component 49 .

\section{Modulation of polysynaptic pathways}

Although phasic locomotor-related presynaptic inhibition has been described in lamprey ${ }^{50}$ and in cat ${ }^{35}$, the mechanisms by which reflex reversal occur are not yet known in vertebrates. In parallel to presynaptic modulation of proprioceptive inputs during fictive locomotion, reflex reversal involves changes of the polysynaptic pathway INs. This level is much more accessible in invertebrates; hence most of the data described below are from studies on invertebrates.

In insects $^{2}$, non-spiking INs are local interneurons that are involved in the polysynaptic pathways from proprioceptors to MNs. Such pathways were characterized first in the locust (Fig. 4A, see ref. 2 for a review). Their participation in reflex reversal was studied primarily in the stick insect ${ }^{26,51,52}$, where it was shown that the sign of the reflex is the result of a balance between excitatory and inhibitory non-spiking INs (NSIs represented in orange in Figure 4B). The level of activity of each type of non-spiking IN is dependent on the "state" of the preparation, that is, the motor program being engaged. In addition to these central control pathways, in the locust ${ }^{21}$ and in the stick insect ${ }^{52}$, some non-spiking INs are monosynaptically excited and/or disynaptically inhibited by proprioceptive inputs. For example, in the stick insect ${ }^{52}$, spiking interneurons (SINs represented in yellow in figure 4B), which are activated by proprioceptive afferents, inhibit the non-spiking interneurons. Such pathways may serve to regulate the positive feedback reflex. When an active movement 
occurs, positive feedback would automatically increase the excitation of the MNs that command this movement, whose velocity would thus increase continuously. But, at this stage, the presence of the disynaptic inhibitory pathway, which conveys velocity information, would limit the velocity of movement. In crayfish ${ }^{27}$ (Fig. 4C), during the assistance reflex, a group of non-spiking INs, the ARINs (assistance reflex interneurons), receive monosynaptic EPSPs from CBCO neurons, and directly connect to the MNs that will help the ongoing movement (positive feedback). ARINs are strongly activated by movement-sensitive CBCO neurons. Furthermore, at least in the case of the ARINs that reinforce depressor MN activity during downward movements of the leg, up to 8 velocity-coding downward movement-sensitive CBCO sensory neurons converge onto a single ARIN. However, without any regulatory system, such positive feedback could have dangerous consequences because it would engender instability. The existence of such a regulatory system is indicated by the following observation : whereas low velocity $(0.05 \mathrm{~mm} / \mathrm{s})$ movements imposed on the CBCO evoke only compound EPSPs in ARINs, during high velocity $(0.25 \mathrm{~m} / \mathrm{s})$ movements, the excitatory response is rapidly blocked by a compound IPSP. The inhibition observed in ARINs has been attributed to an assistance reflex-controlling IN ( $\left.\mathrm{ARCIN}^{27}\right)$. ARCINs are highly dependent on the velocity of joint movement: the faster the movement, the more strongly they inhibit the ARINs. This gain control mechanism could play an essential role in preventing the positive feedback loop from "exploding".

In the cat, polysynaptic pathways also exist in parallel to monosynaptic connections between group I afferent fibers and motoneurons (Fig. 4D). During locomotion, the stance phase could be facilitated by spindle and tendon organ afferents of extensors via three pathways $^{53}$ : a monosynaptic pathway from group I afferent fibers, a disynaptic pathway from group Ia and Ib afferents, and a polysynaptic pathway via the extensor half-center from group Ia and Ib afferents. At the same time, spindle afferents from flexor muscles are activated during the stance phase, and consequently shorten this phase, most likely by inhibiting activity in the extensor half-center ${ }^{5}$. 
In contrast to the simple cases presented above for both invertebrate and vertebrate sensory-motor synapses, interactions between sensory terminals and postsynaptic MNs may be very complex. This is the case for the TCMRO ${ }^{16}$ (Fig. 4E), a crustacean proprioceptive organ consisting of two non-spiking sensory neurons, a static (S) fiber and a dynamic (T) fiber, which are stretched when the leg moves backward (remotion). At rest, the negative feedback reflex is due primarily to the $T$ fiber activating promotor (Pro) MNs monosynaptically during remotion. During rhythmic activity, a reversal of the reflex occurs, and the T fiber excites remotor MNs during remotion. Note that within the group of Pro MNs, some are excited by the T fiber while others are inhibited (probably via an IN). Therefore, the sign of the reflex depends on the balance between excitatory and inhibitory influences produced by $\mathrm{T}$ fibers onto Pro MNs, a situation that resembles that described for non-spiking interneurons in insects (Fig. 4A, 4B).

\section{Implication of MNs in the regulation of proprioceptive reflexes}

Are MNs passive output elements or do they actively participate in shaping the reflex response? An increasing number of data in both vertebrates and invertebrates indicate that MNs (1) may display active membrane properties, and (2) make output connections onto other neurons in the central network.

Active properties in MNs result from the existence of voltage-dependent conductances in their membranes. However, such non-linear and oscillatory membrane properties of MNs are rarely spontaneously expressed ${ }^{54}$, but are, in most cases, seen only in the presence of neuromodulators such as serotonin ${ }^{55,56}, \mathrm{NMDA}^{57}$ or muscarinic agonists of acetylcholine ${ }^{29}$. For example, in vertebrates, NMDA-induced TTX-resistant voltage oscillations in the membrane potential of MNs have been observed in lamprey ${ }^{57}$, Rana tadpole ${ }^{58}$, neonatal rat $^{59}$, and turtle ${ }^{60}$ MNs. Thus the status of MNs in at least some vertebrate groups is not very different from that previously shown to exist in many invertebrate motor networks (see Ref 61 for a review). 
In crayfish, we have shown that active membrane properties of MNs are involved in the reversal of the reflex. Indeed, the reflex reversal is not a simple sign inversion of the sensory-motor pathways. Three main changes are observed: (1) the firing frequency within bursts is substantially increased; (2) the relationship between $\mathrm{MN}$ bursts and imposed movements is less precise; (3) some previously active MNs become silent, while previously silent MNs may become active. In the preceding paragraphs, we have shown that both presynaptic inhibition of primary afferent and changes in the activation level of polysynaptic pathways are involved in reflex reversal. In addition, a large part of the observed changes in motor output is due to changes in $\mathrm{MN}$ properties.

When the locomotor generator is activated, the MNs themselves play a role in the suppression of the resistance reflex and the increased activity of MNs in the assistance response. At the same time that presynaptic inhibition blocks the negative feedback pathways, other branches or other proprioceptive afferents activate polysynaptic positive feedback pathways via interneurons. Moreover, some of the MNs involved in this positive feedback now express active plateau properties, which can likewise be induced by muscarinic agonists of acetylcholine $^{29}$ (compare Fig. 5A with Fig. 5C). Consequently, the intensity of discharge of depressor MNs is much higher than it is during resistance reflex responses evoked at rest (compare Fig. 5B with Fig. 5D). Direct reciprocal inhibition between antagonistic MNs ${ }^{62,63}$ would then result in the more depolarized group alone being active, while the antagonistic one is inhibited (Fig. 5E). Since the polysynaptic pathways involved in the assistance reflex response produce larger EPSPs in MNs than does the monosynaptic resistance reflex pathways (partly because of presynaptic inhibition in the monosynaptic resistance reflex pathway), only MNs involved in the assistance reflex response will be allowed to fire. They thus will massively inhibit the antagonistic MNs. In this situation, the active properties in MNs are partly responsible for the massive blocking of the resistance reflex responses.

In addition to the existence of active membrane properties, MNs in most vertebrate and invertebrate systems (except insects) share another characteristic of neurons involved in central pattern generation: the possibility of influencing other neurons of the central network through output synapses. The recurrent inhibition mediated by the Renshaw cell constitutes a well-known example of an output synapse from a MN onto central neurons in mammals (Fig. 6A). It is now known that the Renshaw cells inhibit Ia interneurons, thereby allowing the 
MNs to exert control over the segmental sensory-motor pathways of the reciprocal inhibition circuit $^{64}$. However, Renshaw cells are also controlled by descending excitatory and inhibitory inputs that could, thereby, adjust the excitability of all MNs around a joint. The most spectacular example of a MN controlling its presynaptic neuron terminals was demonstrated in the stomatogastric system of the $\mathrm{crab}^{65}$ (Fig. 6B). A MN named LG (lateral gastric) makes an inhibitory synapse and an electrical synapse onto a presynaptic terminal (SNAX) of an interneuron (modulatory commissural neuron 1: MCN1). When MCN1 fires tonically, it induces rhythmic activity in post-synaptic neurons. However, the terminal branches of MCN1 behave very differently from the remainder of the neuron. The synapses between LG and MCN1, together with the membrane properties of the LG neuron and its reciprocal inhibition with Int1(interneuron 1), transforms MCN1's tonic firing into a bursting activity of the terminal that then entrains the other elements of the network. It is interesting to note that in this case, a part of a neuron (the SNAX terminal) is used by a post-synaptic MN to generate a rhythmic activity in the gastric network. Such functioning principles would be difficult to decipher in more complex systems because they suppose to consider the neuron not as a whole, but rather to treat separately some of its compartments which may not be accessible to recording techniques.

If we now consider the MNs involved in locomotion in crayfish, we note that, similarly, MNs can exert a direct inhibitory control over their proprioceptive afferents ${ }^{66}$ (Fig. 6C). Such a mechanism has not yet been described in other sensory-motor systems. In intracellular recordings from CBCO terminals, two types of PADs are observed during fictive locomotion. In addition to the large GABA-mediated PADs described above, small amplitude slowly developing PADs (sdPADs) that can also be produced by antidromic MN stimulation, are also observed. These sdPADs persist in the presence of picrotoxin, and therefore do not involve the classical chloride channel associated with $\mathrm{GABA}_{\mathrm{A}}$ receptors. In contrast, sdPADs are produced by a glutamate receptor that activates a mixed $\mathrm{K}^{+}$and $\mathrm{Na}^{+}$conductance with a reversal potential of $-55 \mathrm{mV}$. The decrease in membrane input resistance during the activation of this glutamate receptor indicates that sdPADs exert a presynaptic inhibition on the proprioceptive message by a purely shunting mechanism. In contrast to the gain control mechanism studied in the assistance reflex, the sdPAD gain control is based on the postsynaptic $\mathrm{MN}$ activity, and is activated only when MNs are very active. 
With the exception of the insect, in most locomotor systems, MNs make central electrical connections with other MNs, as was recently demonstrated in the frog ${ }^{67}$. In mammals, widespread electrotonic coupling is transient during development. However, it persists in retina, inferior olive, hippocampus, striatum ${ }^{68}$, neocortex ${ }^{69}$ and in the postnatal life of some spinal MNs. In crayfish, electrotonic connections are a widespread feature and have been extensively studied ${ }^{70}$. As was the case for $\mathrm{CBCO}$ fibers, both anatomical and electrophysiological techniques were used to demonstrate such connections between MNs in crayfish. Depolarizing current injected into one $\mathrm{MN}$ can activate up to $4 \mathrm{MNs}$ of the same functional group. Similarly, Lucifer Yellow injected into one depressor MN reveals a group of 4-5 stained MNs. However, as is the case for CBCO fibers, these electrical connections are heterogeneous among the depressor MN population, the efficacy of the coupling (generally weak and always $<10 \%)$ and the number of MNs connected (0-5) varying between MNs. Consequently, electrical coupling seems to define sub-groups of MNs with synchronized activity. In this way, a single input from a CBCO afferent would tend to propagate to the MNs of the same sub-group. This is likely to be the case during CPG activation.

For reasons of efficacy and adaptability, each of the control mechanisms we have described so far needs to be adapted to the behavioral requirement state of the whole animal, which may change with time due to growth or seasonal rhythms. In the last part of this review, we will consider two slowly modulating mechanisms that allow a regulation of sensory-motor circuit performance over long periods of time.

\section{Long-term modifications of the proprioceptive feedback system}

Neural networks behave as highly non-linear systems, and the integration of proprioceptive feedback into central processing is therefore a dynamic task. In such systems, control parameters need to be fitted in order to adapt neural network machinery to behavioral requirements. These changes operate with a much longer time scale (>hours) than the simple online control mechanisms described above, which generally operate at the millisecond time scale. 
In rat, in monkey and in human, the stretch reflex can be operantly conditioned ${ }^{71-73}$. The site and nature of this plasticity lies in the spinal cord itself ${ }^{9}$. In contrast to long-term depression, which seems to result directly from changes in the MN properties, long-term potentiation (LTP) likely results from a decrease in the disynaptic inhibition involving group-I afferent fibers 65 .

In invertebrates, LTP exists in motor systems too, as was demonstrated between MNs involved in the jump in the locust ${ }^{74}$. More recently, in the crayfish, LTP of synapses between $\mathrm{CBCO}$ afferents and $\mathrm{MNs}$ has been demonstrated ${ }^{10}$. In contrast to the plastic changes reported above, this LTP is intrinsic to the two-neuron (sensory afferent/MN) connection and is initiated by the postsynaptic MN activity (Fig. 6C). The CBCO-MN synapse generally remains quite stable for hours in the absence of postsynaptic activity. However, the intracellular stimulation of the postsynaptic $\mathrm{MN}(10 \mathrm{~Hz}, 5 \mathrm{~min})$ generally induces a significant (up to 300\%) and long-lasting (a few hours; Fig. 5D) increase in the amplitude of the monosynaptic EPSP evoked by the CBCO sensory spike. The mechanisms underlying this LTP have recently been analyzed ${ }^{10}$. It is mediated by glutamate, the MN neurotransmitter in arthropods. A quantal analysis of unitary EPSPs before and after LTP induction demonstrated that the increase in EPSP amplitude results from a large increase in the probability of release from the presynaptic neuron, without changes either in the quantal size or the total number of quanta. This result, associated with the absence of a long-term effect of glutamate upon the MN, strongly suggests that the synaptic changes that lead to LTP are purely presynaptic. Moreover, the results of pharmacological studies indicate that a glutamate metabotropic receptor located on the presynaptic sensory neuron terminal is likely to be involved. Note that in this system, MNs have two effects on their presynaptic sensory neurons: they presynaptically inhibit them and induce LTP in them. However, those two phenomena support different functions and would occur in different states of the network. Retrograde glutamatergic presynaptic inhibition requires high level of motor activity, and is therefore a "protective" mechanism which limits the activity of the postsynaptic MN. In contrast, LTP seems to be more of an "arousal" process observed only in very quiet motor systems, where, via positive feedback, it permits reinforcement of the input synapses controlling motor activity. Due to this LTP mechanism, when a MN is recruited, its sensory pathways are also 
reinforced. This finding reinforces the idea that sensory-motor units rather than MNs represent the real basic components of motor commands.

\section{Concluding remarks}

The data presented in this short review demonstrate that sensory-motor connections are much more complex than was initially thought. Most of the levels of control reported here are fast regulatory systems, but long-term changes are also present. In the crayfish model, most of the adjusting levels are intrinsic to the locomotor network itself, and therefore are activity-dependent. It is striking that similar mechanisms are used in different animal models. For example, PADs involve a GABA-induced activation of chloride channels, which could signify that presynaptic inhibition of primary afferents is a fundamental feature that appeared very early in evolution. However, substantial differences also exist. For example, in primary afferents of insects, the equilibrium potential for chloride is close to the resting membrane potential, whereas it is much more depolarized in vertebrate and crustacean primary afferents. The role of non-spiking interneurons constitutes another substantial difference between vertebrates and invertebrates. Non-spiking interneurons are very suitable premotor elements because they can exert a graded control over the MNs, and achieve independent local processing in their different branches ${ }^{2}$. Are such elements really absent in the vertebrate spinal cord? Or are they simply not accessible to intracellular recordings? More generally, a particularly interesting question concerns the compartmentalization of neuron processing. For example, it seems that the different branches of vertebrate primary afferents are differentially affected by presynaptic inhibition ${ }^{75}$. Future investigations should answer these questions. In addition, there are still many unanswered questions concerning how the sensory-motor pathways described in the different animal models are controlled by descending interneurons, and, more generally, how sensory-motor pathways operate in a real behavioral context. This question will be an important challenge in the future, as we know that, in vertebrates and in mammals in particular, cephalization has resulted in increased control of local circuits by superior structures, and thus likely masks such intrinsic adjustments. 
Acknowledgments: This work was supported by the Centre National de la Recherche Scientifique (CNRS). The authors thank Dr J. Simmers and P. Dickinson for providing valuable comments on the manuscript. 


\section{Selected references}

1 Lloyd, D.P.C. (1943) J.Neurophysiol. 6, 317-326

2 Burrows, M. (1992) Trends Neurosci. 15, 226-232

3 Blight, A.R. and Llinas, R. (1980) Philos.Trans.R.Soc.Lond.B.Biol.Sci. 290, 219-276

4 El Manira, A., Cattaert, D. and Clarac, F. (1991) J.Comp.Physiol.[A] 168, 337-349

5 Pearson, K.G. and Ramirez, J.-M. (1997) in Neurons, Networks, and Motor Behavior (Stein P.S.G., Grillner S., Selverston A.I. \& Stuart D.G., eds.), pp. 225-235, Bradford Book, The MIT Press

6 Wilson, D.M. (1961) J.exp.Biol. 38, 471-490

7 Stein, P.S.G. et al. (1997) Neurons, networks and behavior, A Bradford Book, The MIT Press

8 Pearson, K.G. (1995) in Neural Control of Movement. (Ferrel W.R. \& Proske U., eds.), Plenum

9 Wolpaw, J.R. (1997) Trends Neurosci. 20, 588-594

10 Le Ray, D. and Cattaert, D. (1999) J.Neurosci. 19, 1473-1483

11 Hulliger, M. (1984) Rev.Physiol.Biochem.Pharmacol. 101, 1-110

12 Skorupski, P. and Bush, B.M.H. (1992) Proc.R.Soc.Lond.B.Biol.Sci. 249, 7-12

13 Eccles, J.C., Eccles, R.M. and Lundberg, A. (1957) J.Physiol.(Lond.) 22-50

14 Scott, J.G. and Mendell, L.M. (1976) J.Neurophysiol. 39, 679-692

15 Le Ray, D., Clarac, F. and Cattaert, D. (1997) J.Neurophysiol. 78, 3133-3143

16 Skorupski, P. (1992) J.Neurophysiol. 67, 664-679

17 Shapovalov, A.I. and Shiriaev, B.I. (1978) Exp.Brain Res. 33, 299-312

18 Burrows, M. (1987) J.Neurosci. 7, 1064-1080

19 Wolf, H. and Büschges, A. (1995) J.Neurophysiol. 73, 1861-1875

20 Kittmann, R., Schmitz, J. and Büschges, A. (1996) J.Neurobiol. 31, 512-532

21 Burrows, M., Laurent, G. and Field, L.H. (1988) J.Neurosci. 8, 3085-3093

22 DiCaprio, R.A. and Clarac, F. (1981) J.exp.Biol. 90, 197-203

23 Duysens, J. et al. (1990) Exp.Brain Res. 82, 351-358

24 Rudomin, P., Romo, R. and Mendel, L.M. (1998) Presynaptic inhibition and neural control, Oxford Univesrity Press

25 Cattaert, D., El Manira, A. and Clarac, F. (1992) J.Neurophysiol. 67, 610-624

26 Büschges, A. and Wolf, H. (1995) J.Neurophysiol. 73, 1843-1859

27 Le Ray, D. and Cattaert, D. (1997) J.Neurophysiol. 77(4), 1963-1978

28 Bush, B.M.H. and Laverack, M.S. (1982) in The biology of crustacea (Atwood H.L. \& Sandeman D.C., eds.), pp. 397-467, Academic Press

29 Cattaert, D. et al. (1994) J.Neurophysiol. 72, 1622-1633

30 Berry, M.S. and Pentreath, V.W. (1976) Brain Res. 105, 1-20

31 Frank, K. and Fuortes, M.G.F. (1957) Fed.Proc.Fed.Am.Soc.Exp.Biol. 16, 39-40

32 El Manira, A. et al. (1993) J.Neurophysiol. 69, 2248-2251

33 Pasztor, V.M. and Bush, B.M.H. (1987) Nature 326, 793-795

34 Rossi-Durand, C. (1993) Brain Res. 632, 1-15

35 Gossard, J.-P., Cabelguen, J.-M. and Rossignol, S. (1991) J.Neurophysiol. 65, 914-926

36 Burrows, M. and Laurent, G. (1993) J.Neurosci. 13, 808-808

37 Rudomin, P. (1990) Trends Neurosci. 13, 499-505

38 Burrows, M. and Matheson, T. (1994) J.Neurosci. 14, 272-282

39 Cattaert, D. and El Manira, A. (1999) J.Neurosci. 19, 6079-6089

40 El Manira, A. and Clarac, F. (1991) Eur.J.Neurosci. 3, 1208-1218

41 Gallagher, J.P., Higashi, H. and Nishi, S. (1978) J.Physiol.(Lond.) 275, 263-282

42 Lamotte, d.I. et al. (1998) J.Comput.Neurosci. 5, 141-156

43 Cattaert, D., El Manira, A. and Clarac, F. (1994) Brain Res. 666, 109-112

44 Bévengut, M., Clarac, F. and Cattaert, D. (1997) J.Neurophysiol. 78, 1180-1183

45 Marder, E. (1998) Curr.Biol. 8, R795-R797

46 Edwards, D.H., Yeh, S.R. and Krasne, F.B. (1998) Proc Natl.Acad.Sci.U.S.A 95, 7145-7150

47 Pearlstein, E., Clarac, F. and Cattaert, D. (1998) Neurosci.Lett. 241, 37-40

48 Büschges, A., Kittmann, R. and Ramirez, J.M. (1993) J.Neurobiol. 24, 598-610 
49 Matheson, T. (1997) J.exp.Biol. 200, 1317-1325

50 El Manira, A., Tegner, J. and Grillner, S. (1997) Eur.J.Neurosci. 9, 696-705

51 Bässler, U. (1993) Br.Res.Rev. 18, 207-226

52 Büschges, A. (1995) Verh.Dtsch.Zool.Ges. 88, 139-151

53 Pearson, K.G. (1995) Curr.opin.Neurobiol. 5, 786-791

54 Hancox, J.C. and Pitman, R.M. (1993) J.exp.Biol. 176, 307-310

55 Kiehn, O. (1991) Trends Neurosci. 14, 68-73

56 Hounsgaard, J. and Kiehn, O. (1989) J.Physiol.(Lond) 414, 265-282

57 Wallen, P. and Grillner, S. (1987) J.Neurosci. 7, 2745-2755

58 Sillar, K.T. and Simmers, A.J. (1994) Eur.J.Morphol. 32, 293-298

59 Hochman, S., Jordan, L.M. and Schmidt, B.J. (1994) J.Neurophysiol. 72, 2559-2562

60 Guertin, P.A. and Hounsgaard, J. (1998) J.Neurophysiol. 80, 3380-3382

61 Selverston, A.I. et al. (1997) in Neurons, Networks, and Motor Behavior (Stein P.S.G., Grillner S., Selverston A.I. \& Stuart D.G., eds.), pp. 105-117, A Bradford Book, The MIT Press

62 Pearlstein, E., Marchand, A.R. and Clarac, F. (1994) Eur.J.Neurosci. 6, 1445-1452

63 Pearlstein, E. et al. (1998) J.Comp.Neurol. 399, 241-254

64 Hultborn, H. and Pierrot-Deseilligny, E. (1979) J.Physiol.(Lond) 297, 267-287

65 Coleman, M.J., Meyrand, P. and Nusbaum, M.P. (1995) Nature 378, 502-505

66 Cattaert, D. and Le Ray, D. (1998) Eur.J.Neurosci. 10, 3737-3746

67 Perrins, R. and Roberts, A. (1995) J.Neurophysiol. 73, 1013-1019

68 O'Donnell, P. and Grace, A.A. (1997) Neuroscience 76, 1-5

69 Nadarajah, B. et al. (1996) J.Comp.Neurol. 376, 326-342

70 Chrachri, A. and Clarac, F. (1989) J.Neurophysiol. 62, 1237-1250

71 Evatt, M.L., Wolf, S.L. and Segal, R.L. (1989) Neurosci.Lett. 105, 350-355

72 Chen, X.Y. and Wolpaw, J.R. (1995) J.Neurophysiol. 73, 411-415

73 Wolf, S.L. et al. (1995) Exp.Brain Res. 107, 96-102

74 Parker, D. (1995) Eur.J.Neurosci. 7, 1097-1106

75 Lomeli, J. et al. (1998) Nature 395, 600-604 


\section{Figure legends}

Fig. 1. Comparison of the organization of the "stretch" reflex in vertebrate and arthropods. (A) In vertebrates, proprioceptors involved in the stretch reflex are spindle muscles, consisting of intrafusal fibers connected to primary afferent neurons. In the two antagonistic muscles represented, a sensory neuron (Ia afferent) monosynaptically excites MNs of the homonymous muscle (F: flexor; E: extensor). When an extension movement is imposed on the joint (green arrow), the flexor muscle and its spindles are stretched. This information is conveyed by the corresponding Ia afferent (blue) that excites the flexor MN (pink). Subsequently this MN commands the contraction of the flexor muscle and opposes the imposed movement (pink arrow). In addition to the excitation of the homonymous MNs, the antagonistic MNs are inhibited (reciprocal inhibition) by a disynaptic pathway involving a Ia inhibitory IN. (B) In arthropods, chordotonal organs are the proprioceptors involved in the "stretch" reflex. In contrast to spindle muscles of vertebrates, chordotonal organs are located outside the skeletal muscles. They consist of an elastic strand (green bar) that crosses the joint. This strand is stretched when the joint opens, and released when the joint closes. In the strand, two populations of sensory neurons (blue and orange circles) code for these two directions of movement. When an extension movement is imposed on the joint (green arrow) only one population of sensory neurons is activated (blue). These sensory neurons monosynaptically activate the MNs of the flexor muscle that opposes the imposed movement.

Fig. 2. Schematic representation of the sensory-motor system controlling the crayfish second leg joint. (A, B) Arrangement of the chordotonal organ (CBCO) at the coxo-basipodite joint (A) commanded by levator (LEV) and depressor (DEP) muscles for upward and downward movements respectively (B). Coxo: coxopodite; Basi: basipodite. (C) In vitro arrangement of the ventral nerve cord of the crayfish, together with the motor and sensory nerves to the $5^{\text {th }}$ leg. The CBCO proprioceptor can be mechanically stimulated to mimic leg movements. (D) Schematic representation of a $\mathrm{CBCO}$ sensory terminal (CBCO term) and a depressor motor neuron (Dep MN) in the $5^{\text {th }}$ thoracic ganglion. (E) Paired intracellular recordings from a $\mathrm{CBCO}$ terminal coding for upward leg movement and depressor MN shows the MN response (EPSP) to the CBCO sensory spike. (F) Responses of proprioceptive neurons and motoneurons to leg movements. At the bottom are schematically represented the two classes of CBCO terminals responding to downward leg movements (CBCO term1) and to upward leg movements (CBCO term2) respectively. The monosynaptic responses of all 12 depressor MNs (successive intracellular recordings in the same experiment, in a high $\mathrm{Ca}^{+2}$ and high $\mathrm{Mg}^{+2}$ saline) during ramp movements imposed on the CBCO strand are presented above. CBCO sensory neurons activated by upward movements monosynaptically activate 8 depressor MNs, while $\mathrm{CBCO}$ sensory neurons activated by downward movements monosynaptically activate one depressor assistance MN. Three depressor MNs are not monosynaptically connected to either group of CBCO neurons. Vertical scale bar is for MN intracellular recordings.

Fig. 3. Sensory processing in primary afferents. (A) During oxotremorine-induced rhythmic activity, monitored by the rhythmic bursts of spikes recorded from the nerve innervating the depressor muscle (Dep n), an intracellular recording from a CBCO terminal displays bursts of primary afferent depolarizations (PADs) time-locked with the depressor bursts. (B) During 
each PAD burst, the amplitude of sensory spikes is reduced, as is the amplitude of the corresponding EPSPs recorded from a postsynaptic levator MN. Paired intracellular recordings from a $\mathrm{CBCO}$ terminal (CBCO term) and a levator $\mathrm{MN}$ were performed. The two superimposed recordings were obtained respectively in the absence $(1$, blue) and in the presence $(2$, orange) of a PAD. The amplitudes of the sensory spike in the CBCO term. and the corresponding EPSP in the levator MN are reduced - compare (2) to (1). In the inset, the EPSPs obtained in these two situations have been enlarged to show more clearly the reduction of EPSP amplitude when a PAD was present in the CBCO term. (C, D) Electrical coupling between CBCO sensory neurons. Two coupled CBCO terminals (CBCO term1, CBCO term2) have been stained with Lucifer yellow (intracellularly injected into only one terminal) and analyzed with the confocal microscope (C). Physiological evidence for electrical coupling is shown (D). Depolarizing and hyperpolarizing current pulses were injected into one terminal and the corresponding responses were recorded in the electrically coupled terminal (term 1 and term 2 are represented in green and magenta respectively). (E) Serotonin enhances the response to stretch movement (down) of an intracellularly recorded $\mathrm{CBCO}$ terminal.

Fig. 4. Comparison of the pathways involved in modulation of the stretch reflex in different animals. (A, B) In the locust (A) and in the stick insect (B), proprioceptive afferents are presynaptically inhibited via primary afferent depolarizations (PADs) by at least two groups of spiking interneurons (SINs) known as PADIs (PAD interneurons - no yet identified). Some PADIs (SINs represented in blue) are activated by other proprioceptive fibers from the same chordotonal organ, and exert an online automatic gain control; other PADIs (SINs represented in red) are activated by the central pattern generator (CPG represented in pink) through unknown pathways (dashed line), and are involved in the phasic modulation of the sensory-motor synapse during the walking cycle. In the locust (A), parallel monosynaptic and disynaptic (via a non-spiking IN) pathways from proprioceptive afferents coding for the extension of the tibia activate the flexor MNs (resistance reflex). As is the case in vertebrates, the antagonistic MNs (extensors) are inhibited while the muscles that oppose the imposed movement (flexors) are activated. However, in the case of the locust, this inhibition is achieved via at least four pathways involving spiking (SINs) and non-spiking INs (NSIs). In the stick insect (B), in parallel with monosynaptic connections, flexion sensitive proprioceptive neurons are involved in polysynaptic pathways via a group of non-spiking interneurons (NSI) that make excitatory and inhibitory connections on extensor MNs (Ext. MNs). Depending on the balance between these two effects (controlled by the CPG), a positive or a negative feedback is elicited. A third class of SINs (represented in yellow) regulates the gain of the disynaptic pathway (adapted from Ref. 52 ). (C) In crayfish, proprioceptive afferents are presynaptically inhibited by a type of SINs (represented in red), the PADIs (not yet identified) that exert a phasic presynaptic modulation on the sensory-motor synapse during the walking cycle. The organization of the sensory-motor pathway involves monosynaptic excitatory connections supporting negative feedback (levation of the leg activates depressor MNs), whereas the reversal of the reflex (levation of the leg activates levator MNs) involves disynaptic pathways via non-spiking interneurons (NSIs = assistance reflex interneurons: ARINs). In addition, spiking interneurons (SINs represented in yellow) named ARCINs (assistance reflex controlling interneurons) and activated by velocity-coding proprioceptive neurons inhibit the NSIs of the positive feedback circuit and thus prevent the positive feedback reflex from becoming instable. (D) In the cat, Ia afferents from muscle spindles of the extensor muscle make monosynaptic excitatory contacts on the extensor MNs. However, polysynaptic pathways are involved during stepping in the regulation of stance to swing phases. Feedback from spindle and tendon organ afferents 
of extensors facilitates extensor activities via at least three pathways. Some are monosynaptic, others are disynaptic, and yet others are polysynaptic via the extensor half-center (adapted from Ref. 5) (E) Illustration of complex relations that exist between TCMRO (thoraco-coxal muscle receptor organ) (composed of a $\mathrm{T}$ fiber and a $\mathrm{S}$ fiber with different coding properties), and MNs (Pro: promotor; Rem: remotor) controlling the first leg joint during backward movements in crayfish (adapted from Ref. 16). Filled circles: inhibitory connections; open triangles: excitatory connections; resistor symbols: electrical connections; diode symbols: rectifying electrical synapse; dashed lines: assumed pathways not yet identified.

Fig. 5. Changes in the activity of MNs induced by muscarinic agonists. (A, B) In the absence of muscarinic activation, MNs display passive electrical properties: the $\mathrm{MN}$ depolarizes in response to a pulse of depolarizing current, and repolarizes as soon as the current injection ceases (A). In this condition the $\mathrm{MN}$ displays small monosynaptic responses to electrical stimulation of the CBCO nerve (CBn stim) (B). (C,D) When perfused with muscarinic agonist (Oxo $10^{-5} \mathrm{M}$ ), plateau properties are induced in MNs (C) : a pulse of depolarizing current evokes a depolarization of the $\mathrm{MN}$ that persists after the current pulse (plateau potential); the plateau can be stopped by injection of a pulse of hyperpolarizing current. In this condition, the reflex responses to $\mathrm{CBn}$ stimulation are much larger due to plateau properties (D). (E) schematic diagram illustrating the contribution of plateau properties and reciprocal inhibition to the blocking of the resistance reflex pathways during rhythmic activities (see text for explanations).

Fig. 6. Retrocontrol of afferent message by postsynaptic motoneurons. (A) In mammals, Renshaw cells (RC) exert an inhibitory control on Ia interneurons of the segmental polysynaptic sensory-motor pathways responsible for reciprocal inhibition (adapted from Ref. 64). Two antagonistic MNs, each with its Ia afferent, are represented. Reciprocal inhibition is achieved via Ia interneurons (for clarity only one of the pathways has been represented). The Renshaw cell, activated by a MN, is responsible for the recurrent inhibition of this same MN. In addition, the Renshaw cell inhibits the Ia inhibitory interneuron that synapses on the antagonistic MN. (B) In the stomatogastric system of the crab, MCN1 (modulatory commissural neuron 1) activates LG (lateral gastric neuron from the gastric network) and DG (dorsal gastric neuron) via slow chemical excitatory synapses, and int1 (interneuron 1) via a fast chemical excitatory synapse. In turn, LG inhibits the stomatogastric nerve axon (SNAX) terminals of MCN1, to which it is also electrically coupled (adapted from Ref. 65). (C) In the crayfish walking network, MNs exert a dual control on their CBCO sensory afferents. They elicit shunting presynaptic inhibition of the sensory terminal by activating a glutamate-receptor channel (filled circle), and a long term potentiation of the same sensory afferent via a metabotropic glutamate receptor (parallel T-bars). (D) Long-term potentiation (LTP) of the CBCO-MN synapse induced by activation of the post-synaptic MN (vertical gray bar). In this experiment, intracellular recordings of a $\mathrm{CBCO}$ sensory terminal and a postsynaptic MN were performed simultaneously. The time course of LTP is expressed as relative EPSP mean amplitude (each point represents an average over 5 minutes : Mean + SEM). After the activation of the MN (injection of $10 \mathrm{~Hz}$ depolarizing pulses eliciting two spikes each, for 10 minutes - see top inset "induction"), the EPSP amplitude was increased dramatically. Left and right insets display paired intracellular recordings of the CBCO terminal and the postsynaptic MN before and after LTP induction respectively. Each inset shows 8 superimposed traces. Data on the graph are from a single trial. 
07/11/2019 
Clarac, Cattaert and Le Ray, Fig. 1

A

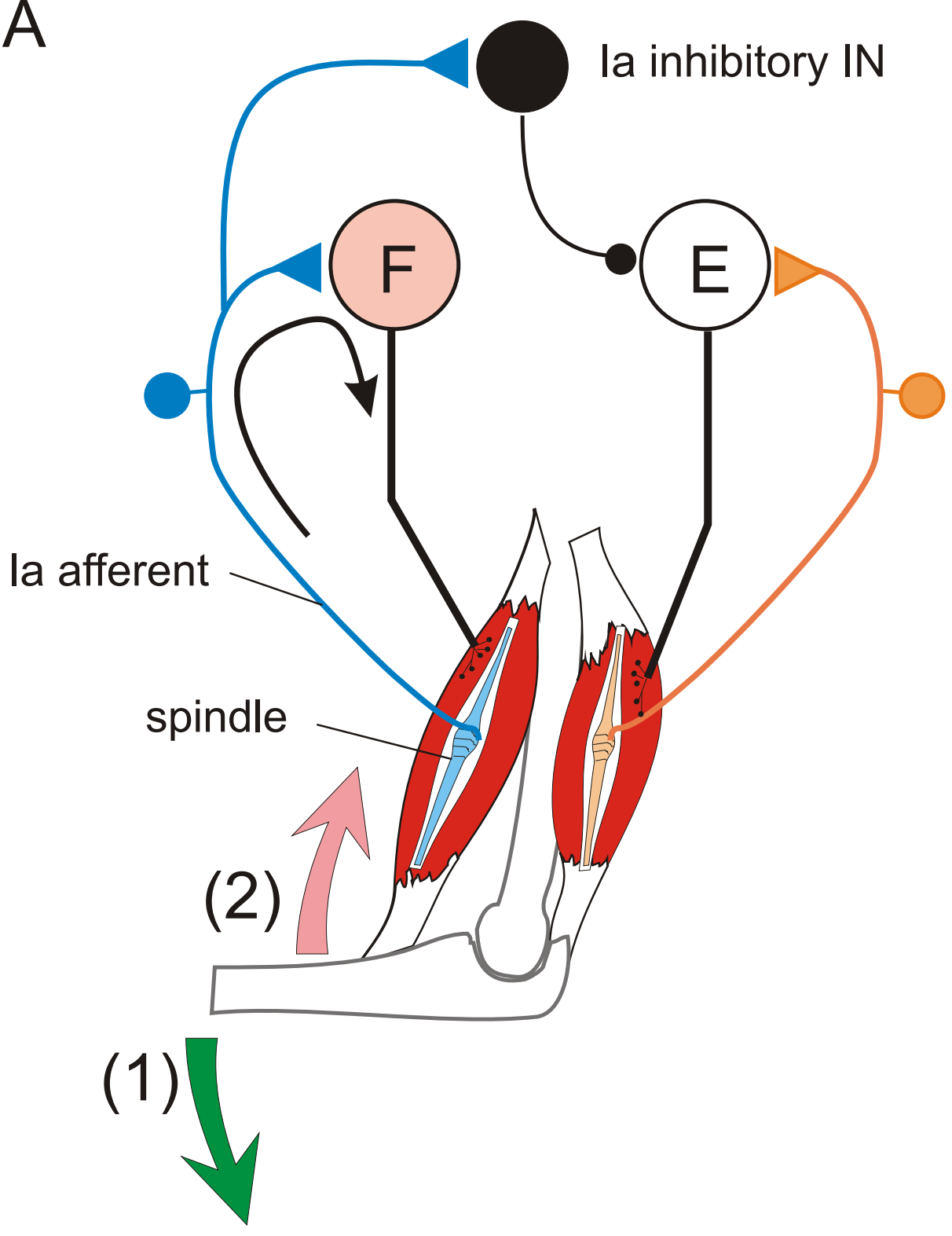

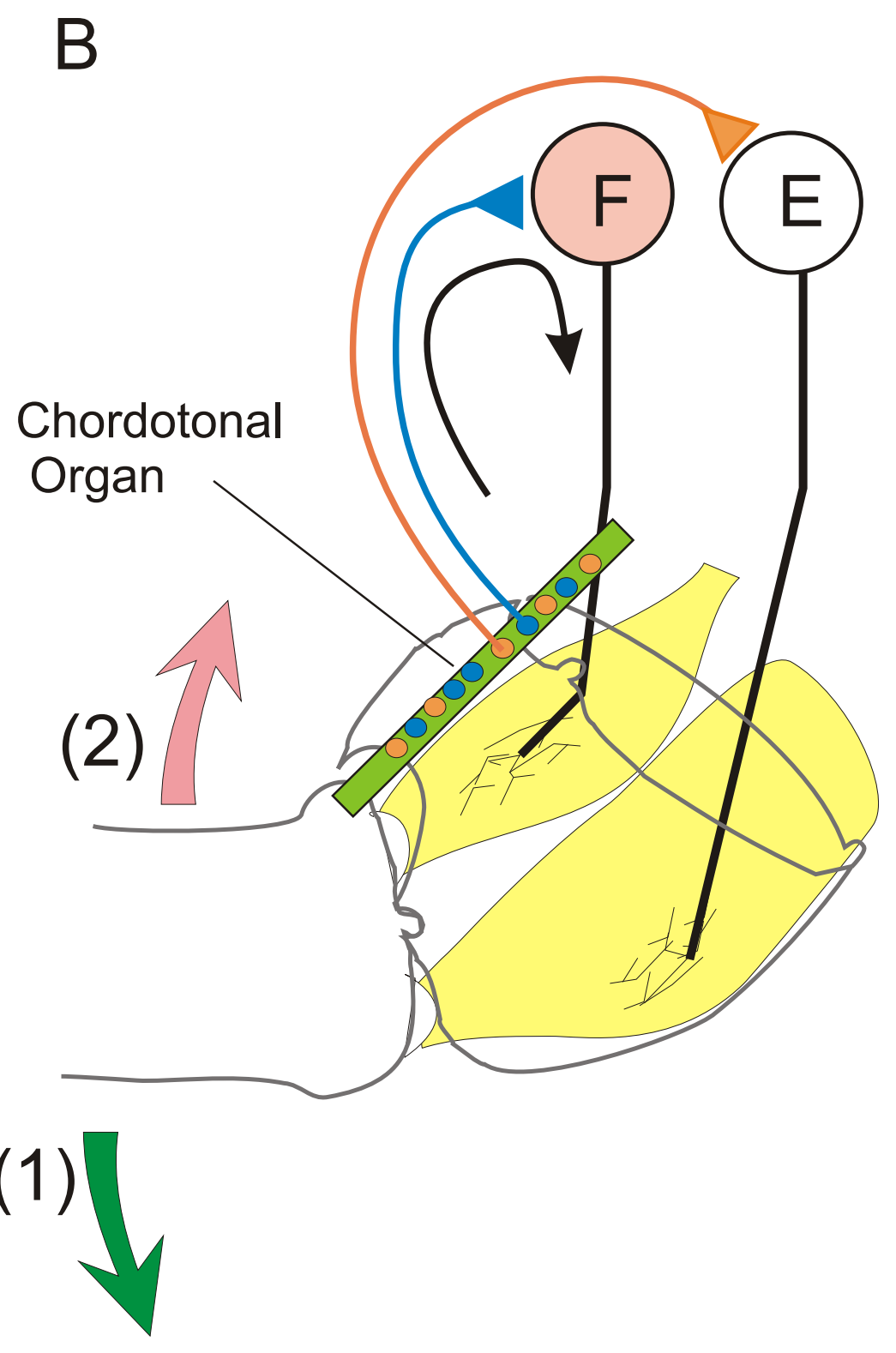


Clarac, Cattaert and Le Ray, Fig. 2
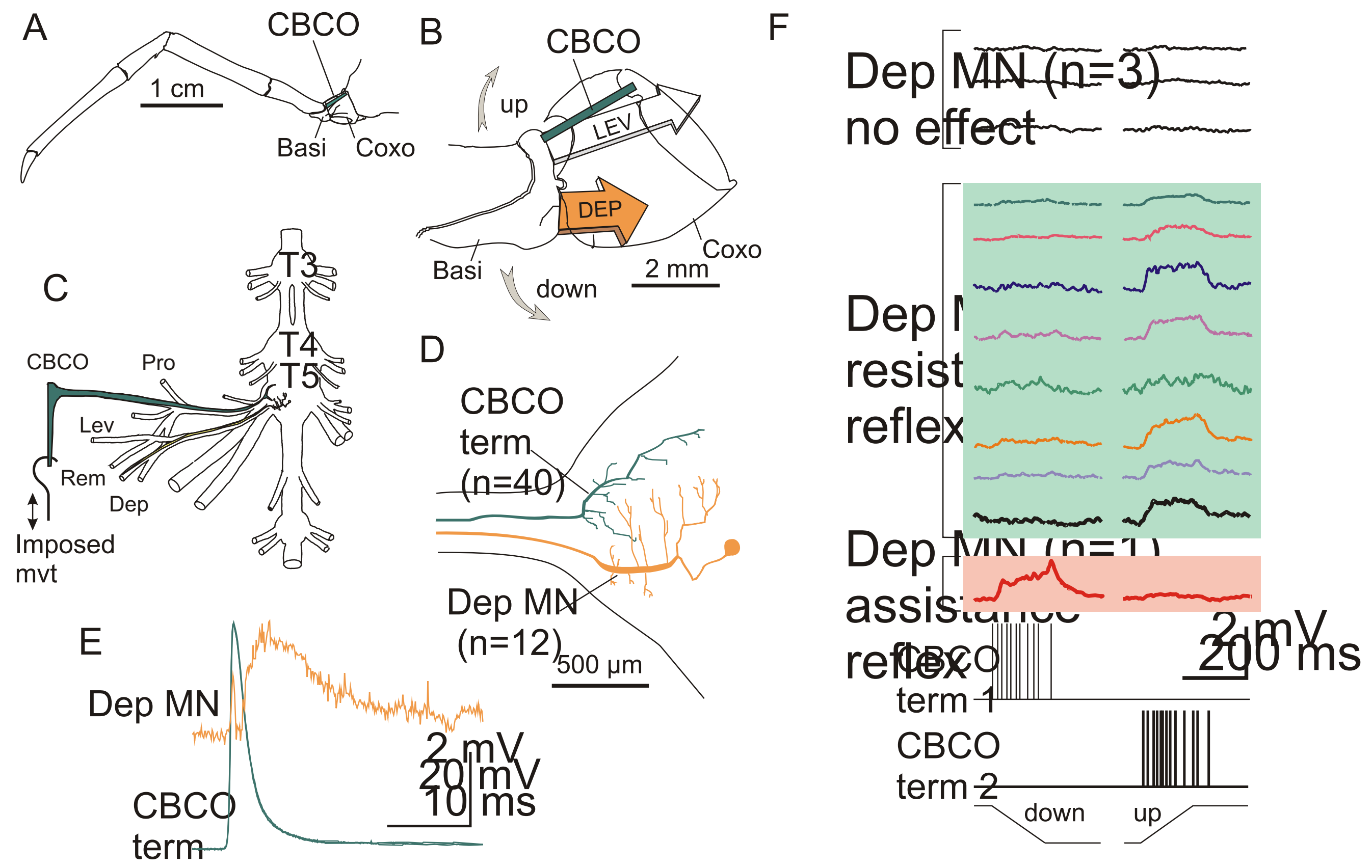
Clarac, Cattaert and Le

A

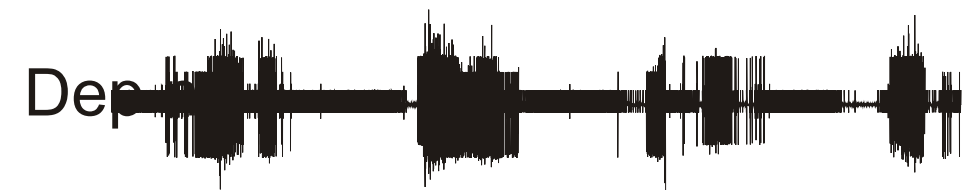

CBCD term,

PADs

$$
\text { PADs }
$$

C

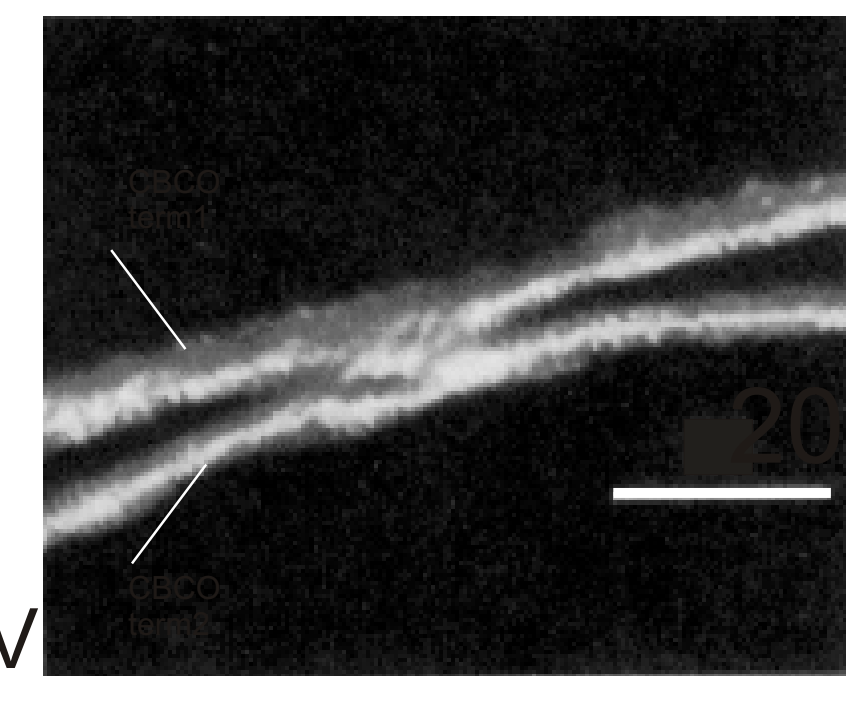

B

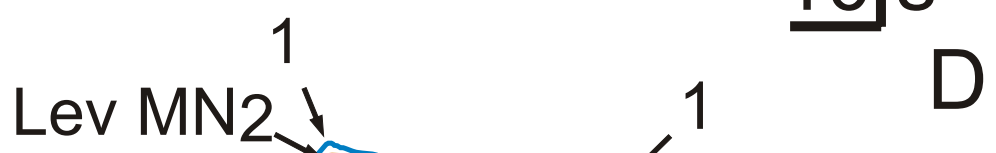

$+5 \mathrm{nA}$ in

CBCO term 1
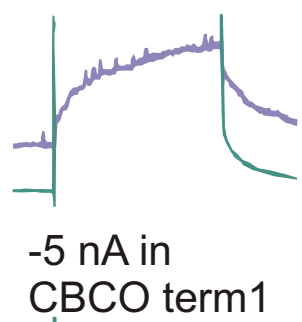

E

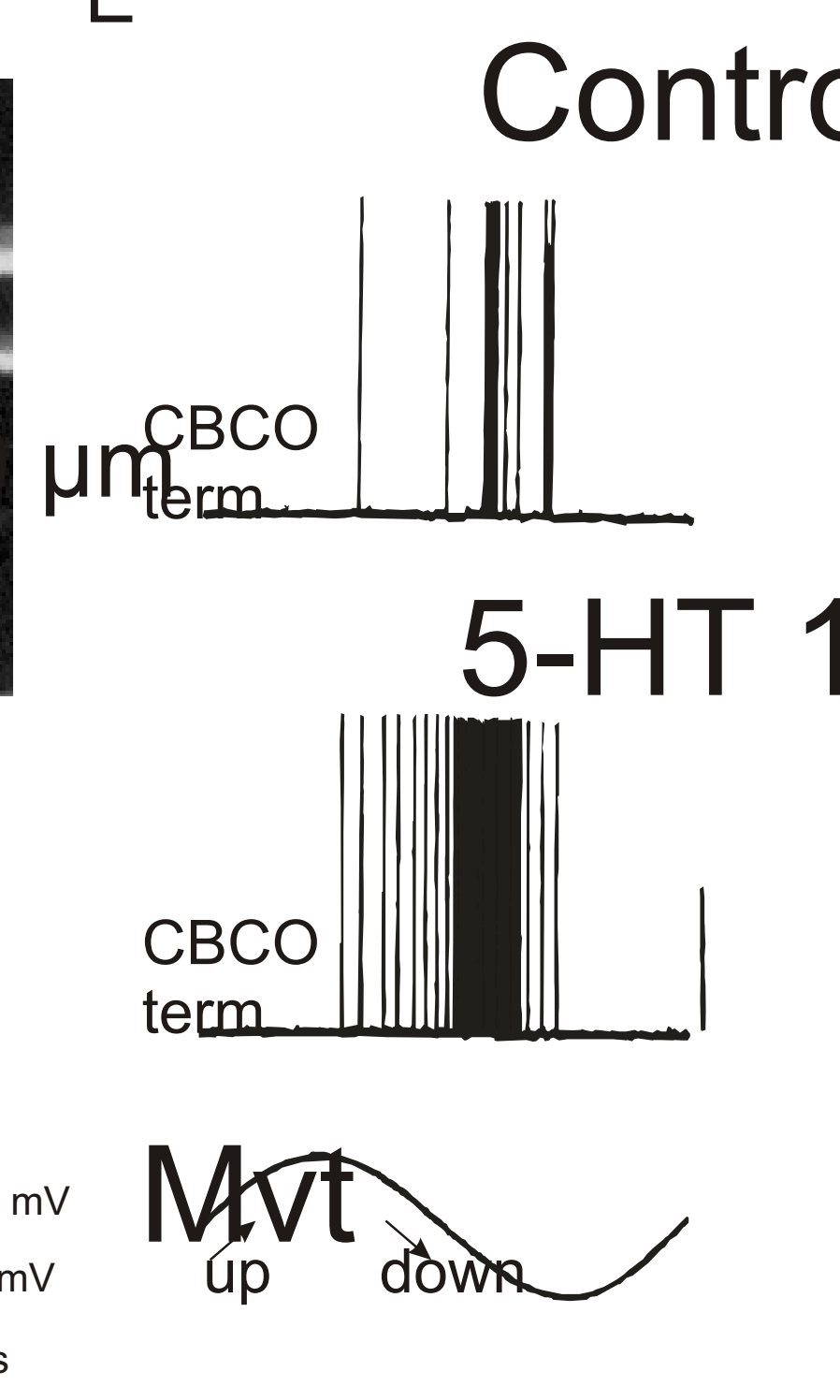




\section{Clarac, Cattaert and}
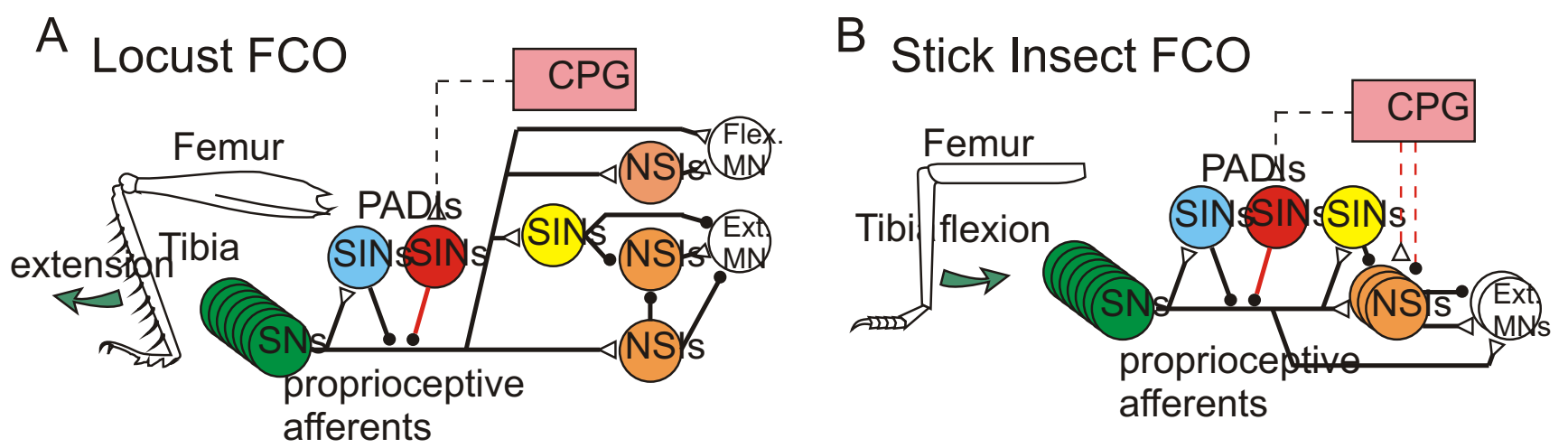

C Crayfish CBCO

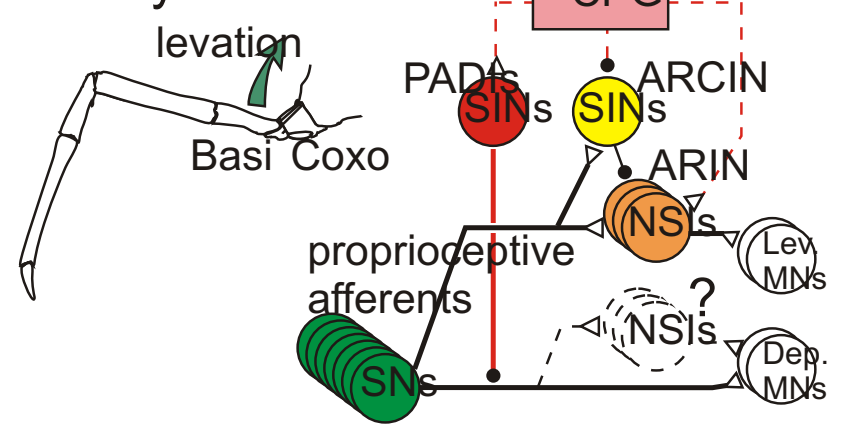

E Crayfish TCMRQ

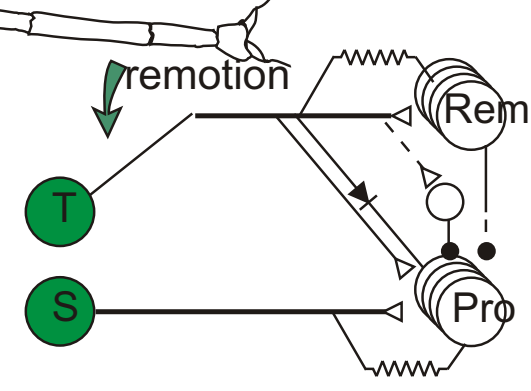

D Cat muscle afferents

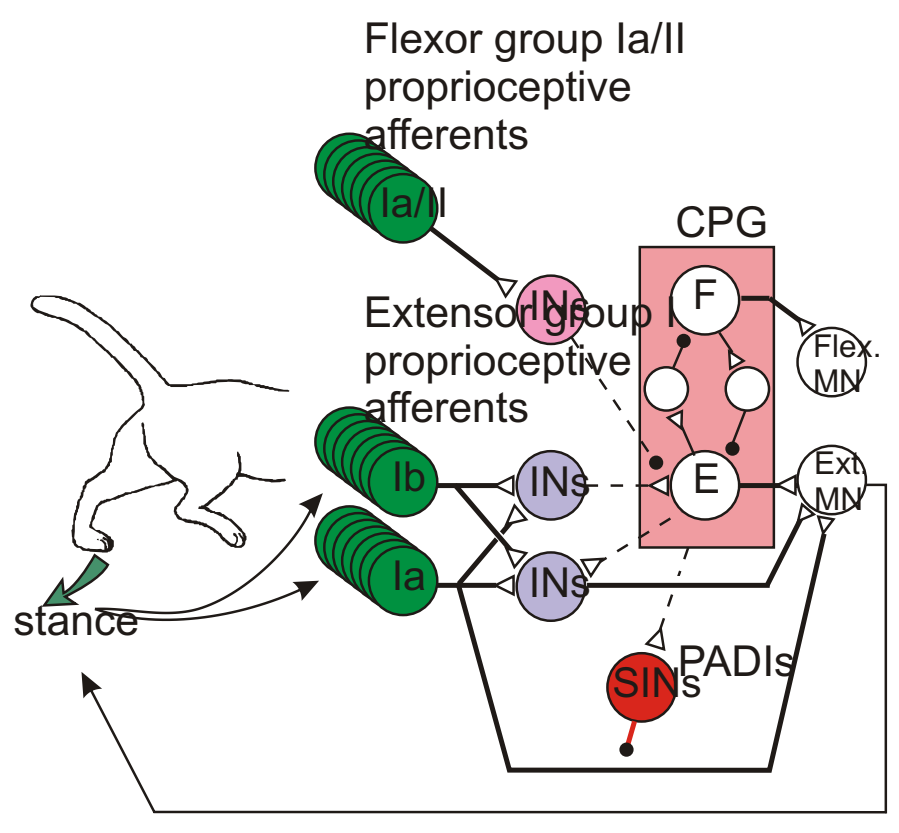




\section{Clarac, Cattaert and L}

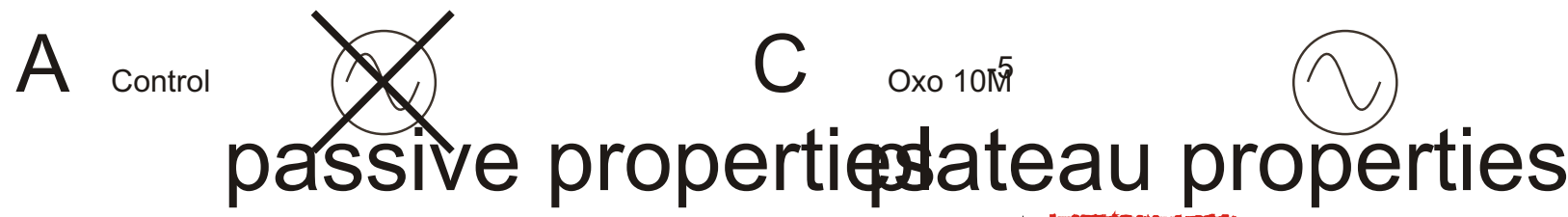
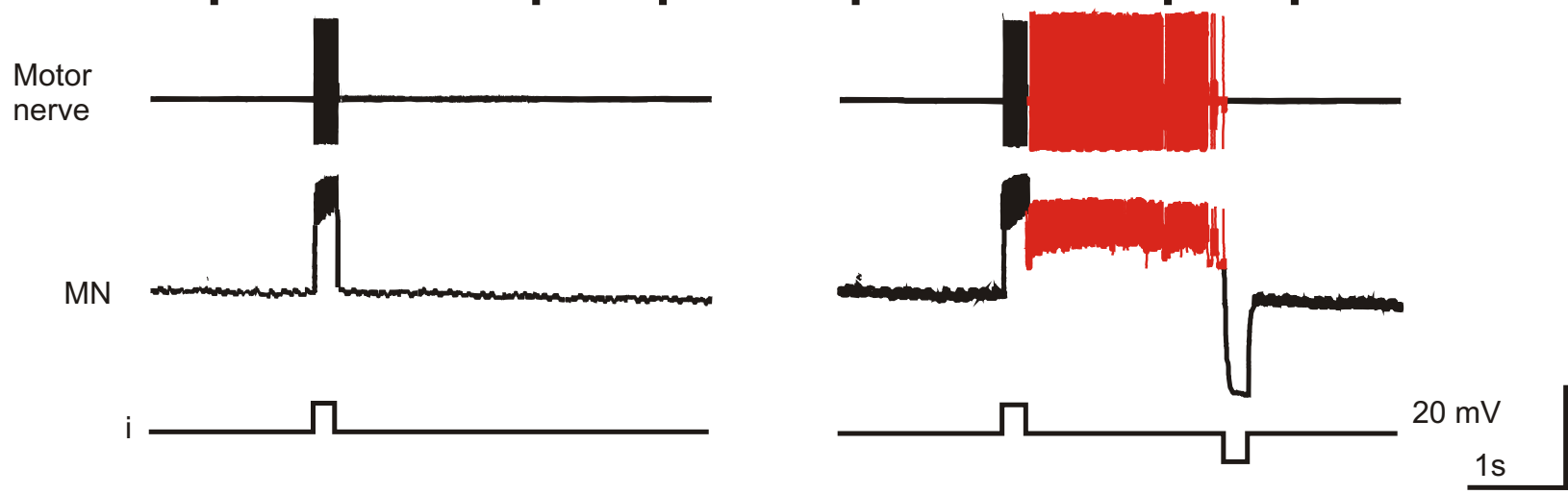

B small reflex respon

MN
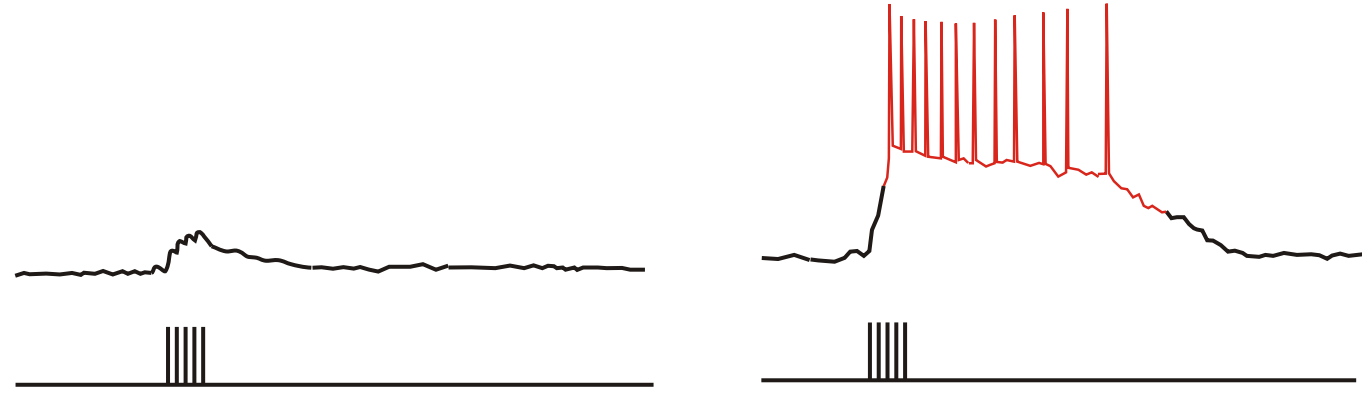

$10 \mathrm{mV}$ $200 \mathrm{~ms}$

$E$

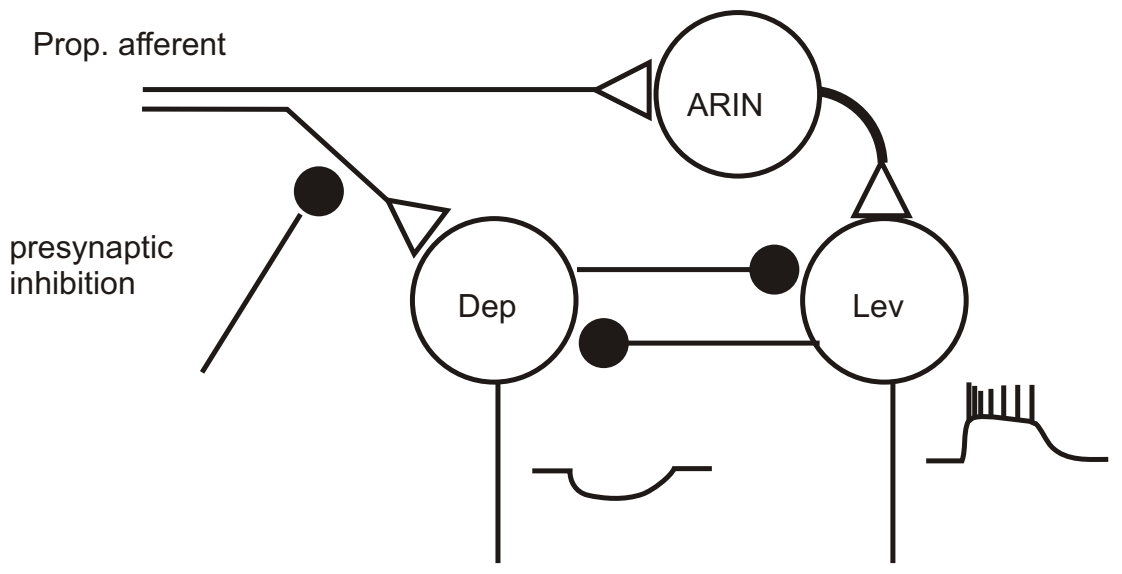




\section{Clarac, Cattaert and L}

A

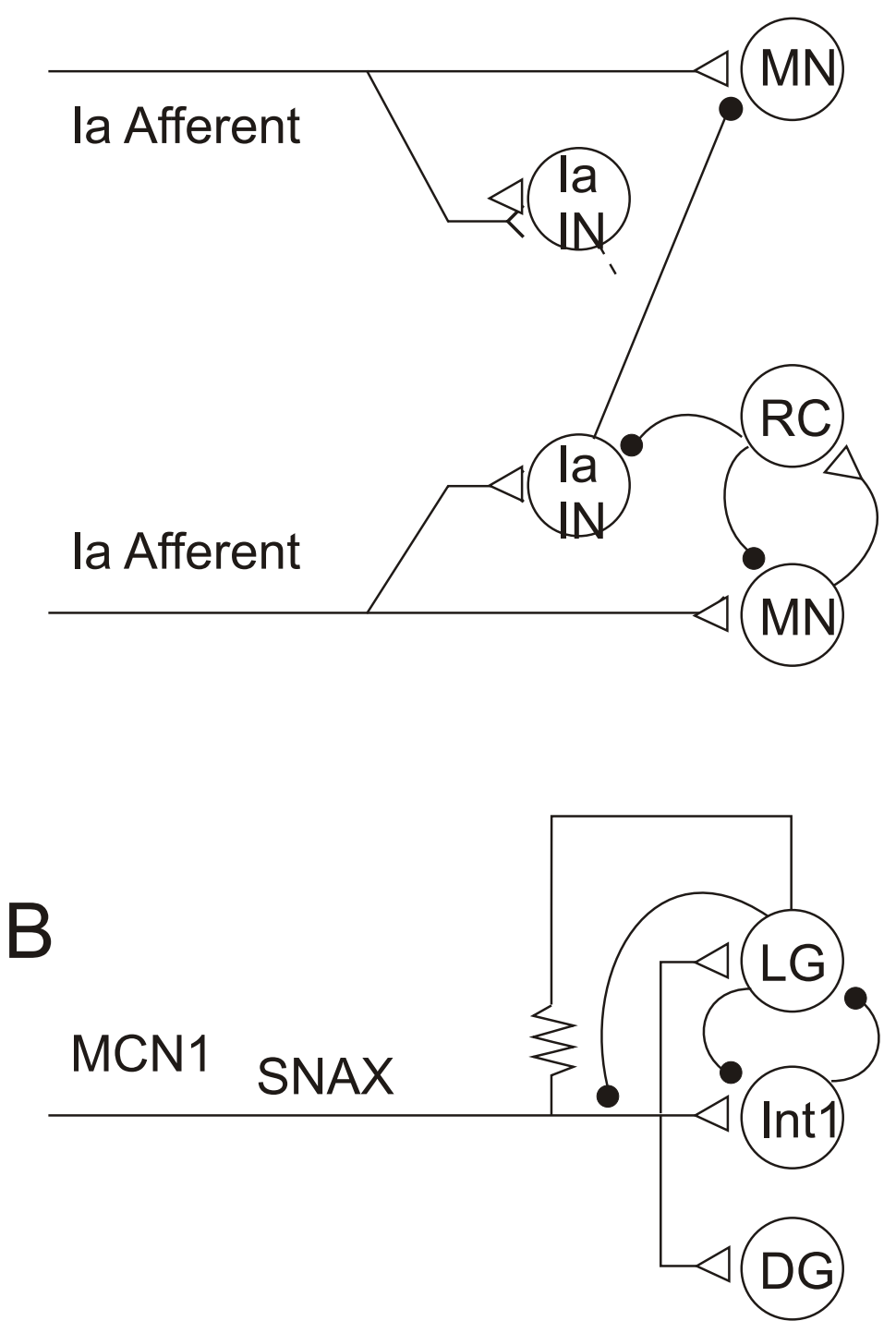

C

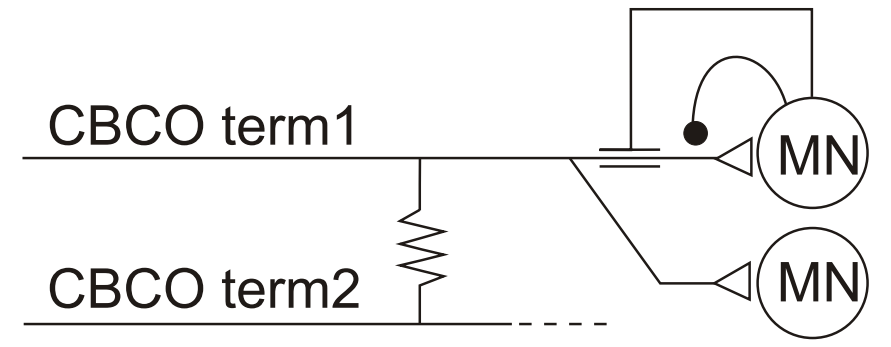

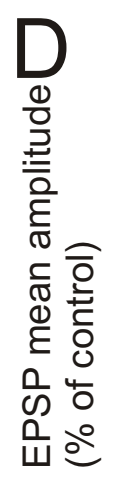

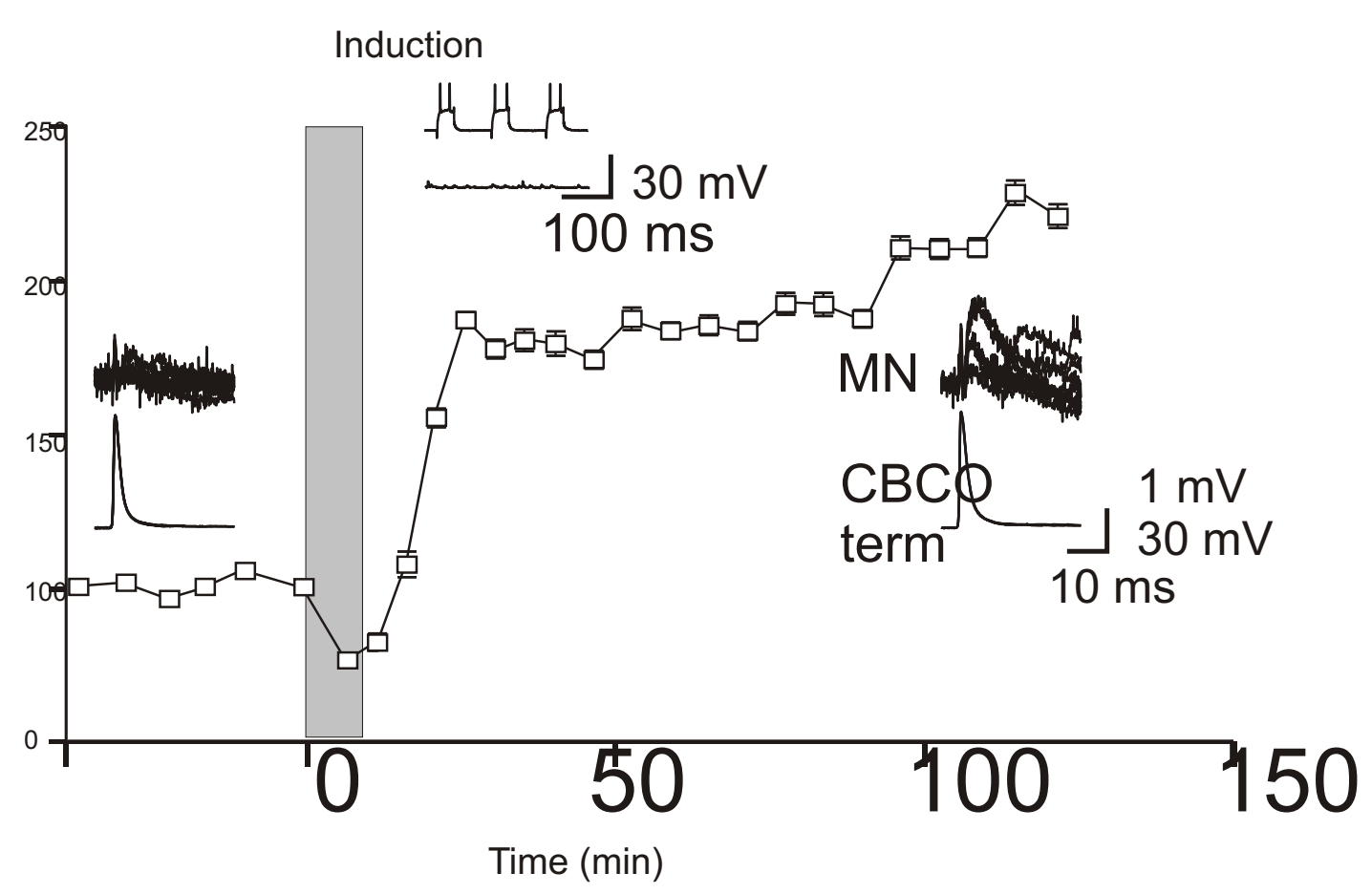

